\title{
Restoration scaling of environmental damages in the face of a changing environment and uncertainty
}

\author{
D. A. Hanson ${ }^{1}$, E. M. Britney ${ }^{2}$, T. G. Stewart ${ }^{3}$, A. W. Wolfson ${ }^{4}$ \\ \& M. Baker ${ }^{5}$ \\ ${ }^{1}$ Hanson Resource Management, Sammamish, WA, USA \\ ${ }^{2}$ ICF International, Seattle, WA, USA \\ ${ }^{3}$ ICF International, Sacramento, CA, USA \\ ${ }^{4}$ Biomass Options, LLC, Portland, OR, USA \\ ${ }^{5}$ US National Oceanographic and Atmospheric Administration, \\ Seattle, WA, USA
}

\begin{abstract}
There is growing recognition that climate change, habitat fragmentation, and other global stressors are altering ecosystem dynamics. This paper discusses the incorporation of dynamic environmental conditions (i.e., non-stationarity) into restoration planning. The context is natural resource damage assessments (NRDA) addressing environmental impacts and lost services, primarily by using habitat equivalency analysis (HEA). Restoration ecologists traditionally incorporate an implicit assumption of stationarity and species-community dynamic equilibrium in designing habitat restoration. HEA has also typically been applied as a deterministic model assuming stationarity of environmental conditions. Anticipated increases in the frequency and severity of environmental disruptions (e.g. high temperatures, drought, extreme precipitation and coastal storm events, changes in the hydrological cycle, increased wildfires, etc.) can alter recovery trajectories, and reset or completely change ecological baselines. Thus, it is beneficial to identify restoration and compensatory actions that explicitly incorporate these influences, provide ecosystem resilience, and thereby protect or enhance primary and compensatory restoration.

Keywords: non-stationarity, natural resources damage assessment, NRDA, habitat equivalency analysis, HEA, ecological restoration, environmental baseline, climate change, restoration portfolios, climate change adaptation, resilience.
\end{abstract}




\section{Introduction}

Ecologists have long recognized that interactions among natural and human stressors are key drivers of ecosystem change. The traditional view has been that despite these drivers of change, most ecosystems are in dynamic equilibrium and fluctuate within an unchanging "envelope of variability" [1]. Restoration ecologists and planners have traditionally incorporated an implicit assumption of stationarity when planning and designing habitat restoration. However, there is growing recognition that anthropogenic climate change, habitat fragmentation, and other global stressors are altering the variability of indicators of ecosystem structures and functions and fundamentally altering ecosystem dynamics and the assumption of stationarity. In some cases, the mean level of population fluctuations change with time, and some recent studies suggest that as variance increases, the potential for reaching a tipping point resulting in significant regime shifts also increases. These observations point to the need to incorporate the variance in physical and biological conditions in ecosystem assessments and restoration planning. Yet there remains little guidance on how to apply this understanding to restoration planning and management, especially when using restoration planning and scaling tools. This paper's objective is to present concepts on how to adapt Habitat Equivalency Analysis (HEA), and Resource Equivalency Analysis (REA), or how to develop new methods for restoration scaling that recognize and address the challenges of quantifying ecological value in a changing environment. Because we are presenting major concepts rather than detailed examples of method changes, we use the terms HEA or REA for brevity while recognizing the differences between the objectives and applications of these analyses.

\subsection{Stationarity versus non-stationarity}

Stationarity is a statistical concept that assumes statistical properties are constant over time and that parameters can be estimated from the instrumental record. Milly et al. famously declared that because of climate change "stationarity is dead and should no longer serve as a central, default assumption in water resource risk assessment and planning" [1].

Non-stationarity refers to the changing distribution of statistical data over time. In the context of natural systems, non-stationarity characterizes meteorological extremes and their distribution over time and space. These extremes, which can be indications of climate change, have direct, indirect, and long-term effects on species and communities. Non-stationarity results in changes to daily and seasonal meteorological and climate parameters, related oceanographic conditions, and to disturbances that alter environmental baselines. Other environmental influences, which may be indirectly related or unrelated to climate change, can result in non-stationarity, such as introduction of invasive species, disease, severe fires, altered habitat succession, or human development. Scaling compensation using HEA or other existing methods is challenging under 
circumstances of non-stationarity as species and communities will experience a variety of non-stationarity stressors with variable responses.

\subsection{Agencies' response to climate change and non-stationarity}

Public sector agencies and governments recognize the fact that climate change poses an increased risk and requires an assessment of ecosystem vulnerability to design and implement effective management plans. Increasingly these agencies are developing cooperative and integrated approaches that offer the promise of mutual benefits and risk reduction given their economic constraints. For example, in the United States the National Fish, Wildlife, and Plants Climate Adaptation Strategy Partnership [2] identifies goals and actions that are applicable nationwide. Review of this strategy and other materials yielded the following common strategies for responding to climate change and nonstationarity in the context of restoration.

- Conduct an inventory of affected resources.

- Identify regional and local stressors.

- Identify the most vulnerable species, habitats, communities and ecosystem services.

- Identify pertinent reference conditions.

- Form/use existing decision support tools (geospatial, ecological modelling).

- Collaborate scientifically (interagency, intergovernmental, tribal, public).

- Identify priorities considering cost, effectiveness and resilience.

- Develop strategies, tactics and plans.

- Monitor the impacts of restoration on affected resources.

- Evaluate effectiveness of restoration.

\subsection{Intensity and timeframes of changing conditions}

Vermeulen et al. [3] identify incremental, systemic and transformative categories for stressors and associated system changes or responses. Anticipated changes in environmental drivers along this gradient reflect increasing climate velocity, non-stationarity intensity and baseline condition change. Restoration responses to the timeframes associated with these categories depend on the species and communities and the specific types and intensity of non-stationarity stressors. These factors can fundamentally alter restoration response as well as the time until a restored condition is achieved. At an extreme, original communities may not be viable and may be 'replaced' by communities that differ from those originally present. A range of ecological concepts and timescales are pertinent to evaluate changed conditions and to guide restoration plans. These include: evolutionary changes, tipping points, novel ecosystems, no-analog Quaternary and Holocene communities, and insights from paleontology and deep geological time.

Every ecosystem has a geospatial distribution pattern or zone of the optimum where species thrive and reproduce. At the extreme boundary of an ecosystem 
the potential for change is significant and represents an increased vulnerability to climate change. Assessments of baseline or reference conditions often reveal more rapid responses to changing climatic conditions and in the future will likely demonstrate increased rates of change in species populations and associated habitats. Therefore restoration planning will need to factor in these likely changes and develop site specific adaptive management strategies.

\section{HEA}

Under many regulatory programs (e.g., Clean Water Section 404 or Endangered Species Act Section 7), project mitigation and/or compensatory restoration are based on replacement of affected areas on an acre to acre basis with some additional factor (e.g., $3 \mathrm{x}$ ) to account for uncertainty. Tools like HEA, which was developed to support Natural Resource Damage Assessment (NRDA) under the Comprehensive Environmental Response, Compensation, and Liability Act (CERCLA) and the Oil Pollution Act (OPA), recognize not all impacted and restored areas are functionally equivalent and instead focus on restoring quantifiable lost ecosystem services. HEA was developed as a scaling method to evaluate ecosystem service losses due to injury or disturbance and restoration gains over time and space, and is applied as a surrogate for habitat valuation to define compensatory restoration. In recent years HEA has been applied to other perturbations of aquatic and terrestrial systems such as evaluation of impacts from major forest fires [4] and mitigation of new transmission lines [5]. HEA routinely is applied as a deterministic model implicitly assuming stationarity of environmental conditions for establishing baseline conditions and scaling ecosystem losses with ecosystem recovery and restoration gains.

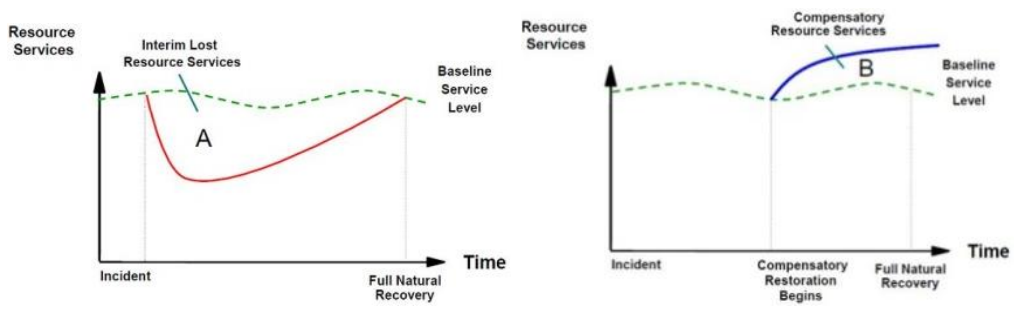

Figure 1: HEA overview.

\subsection{Basic elements}

Figure 1 plots resources services (y axis) over time ( $\mathrm{x}$ axis) and illustrates the basic elements of HEA. In Figure 1A, line 'A' scales the lost ecosystem services as they are reduced by an impact and then return to the baseline condition (restored). Figure 1B shows the compensatory resource services that must be added to make the public whole for the lost use of the service before full recovery. The area under 'B' (i.e., the amount of services) must equal the area under ' $\mathrm{A}$ ' when both service loss and restoration gains are adjusted for the net 
present value to achieve parity. The dashed baseline as depicted in both figures undulates with time reflecting natural, stationarity variation.

\subsection{Climate change implications for HEA}

Baseline data should reflect conditions that would have been expected had the disturbance not occurred, taking into account both natural and anthropogenic processes. Rohr et al. [6] provide an overview regarding how climate change may impact baseline conditions and restoration requirements when using HEA to determine primary and compensatory restoration in response to a hazardous substances release. Baseline and primary restoration scenarios with global climate change can:

- delay recovery/restoration due to interactions with hazardous substances so the initial rate of decline of services is greater;

- $\quad$ reduce the rate of recovery, prolonging the compensatory restoration period;

- $\quad$ prevent services from ever returning pre-injury baseline conditions;

- shift baseline services either higher or lower compared to a scenario of stationarity conditions and thereby accelerate or delay the recovery period;

- increase variance of an ecosystem service that can make it challenging to assess injury and restoration;

- occur in various additional combinations of the aforementioned effects.

\subsection{Implications of expected stressors with unknown timing and impacts}

As depicted in fig. 2 which shows the service loss of expected stressors with unknown timing (modified from Hanson et al. [7]), the uncertainty of an expected, but difficult to predict timing of a perturbation can have a profound impact on restoration scaling under an "all else being equal" assumption.

- Panel A depicts an assumed stable baseline and the theoretical baseline level of services following a severe forest fire. Under this scenario, Area 1 in Panel A depicts the service acre losses.

- Panels B and C depict the same environmental disturbance but demonstrates the effects of different baseline conditions.

- Panel B depicts of a declining pre-fire baseline due to competition-stressed vegetation followed by a stand-changing event at time Tc. The standchanging event could happen at any time along the $\mathrm{x}$ axis unless forest restoration is undertaken (shown at time $\mathrm{Tr}$ ) and/or maintained at sufficient level to reduce stand-changing risk from fire, insect infestation, and disease. Under this scenario, loss from the fire of interest would be equal to discounted Area 1 of Panel B less the discounted Area 2 because ecosystem services after the fire would exceed the ecosystem services under baseline conditions.

- Panel $\mathrm{C}$ acknowledges that, at this point, we have not predicted when the "all else equal" baseline event will occur. Comparing Panels A, B, and C demonstrates that simply agreeing that a fire is likely to occur at some time in the future is insufficient to determining net loss attributable the fire of interest from a more realistic depiction of the future forest condition. 

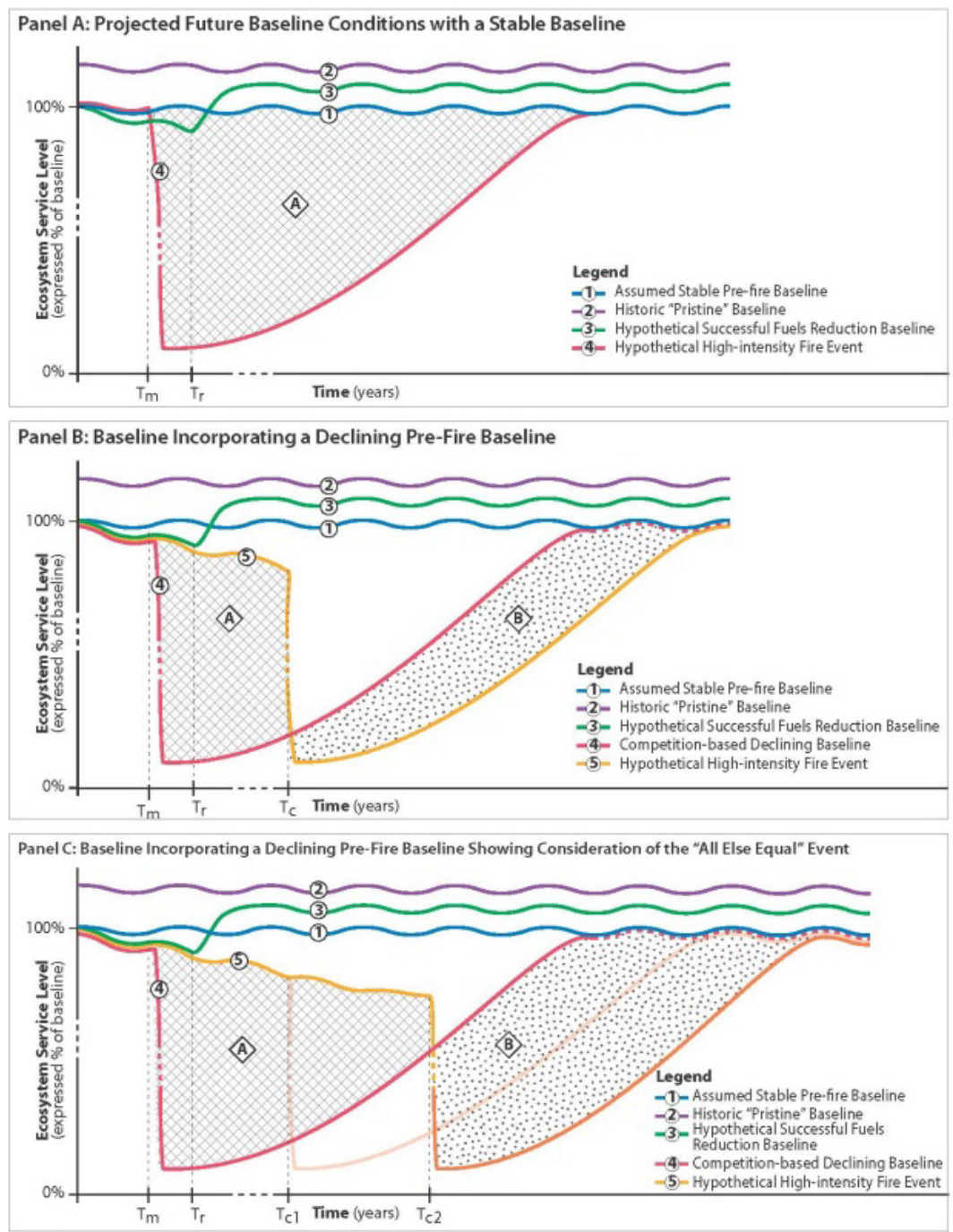

Note: These figures drawn for illustrative purposes only, not to scale.

Figure 2: $\quad$ Service losses.

As stated previously, the service loss impacts and recovery rates depicted in fig. 2 assumed all other conditions beyond the time of the disturbance were equal. This was clearly a simplistic assumption as a fire occurring at a different time, with different weather and soil moisture conditions, could burn at different levels of intensity and severity resulting in different, and potentially beneficial under some circumstances, consequences thereby even further complicating the evaluation of impacts. 


\section{Potential accommodations of HEA}

\subsection{Defining baseline: what was, what would have been, vs. what could be}

Defining the baseline ecosystem services without the evaluated perturbation is often one of the most challenging steps in restoration scaling. Baseline is commonly defined as the ecosystem conditions existing immediately prior to the perturbation of interest (which we define as "what was"). Restoration scaling under this scenario leads to habitat mosaics and connectivity informed by current and/or historic conditions. A more appropriate definition of baseline incorporates either quantitatively, or in some cases qualitatively, how other natural and anthropogenic changes impact baseline conditions. We differentiate a baseline that incorporates considerations of natural and anthropogenic impacts under an assumption of stationarity (which we define as "what would have been") from efforts that seek to also incorporate non-stationarity impacts on baseline (which we define as "what could be"). Actively seeking to understand and address "what could be" incorporates the widest range of influences into the baseline conditions that are sought in a restoration plan and restoration actions.

The concept of sustainability is particularly relevant in assessing the concept of non-stationarity. Without a context for planning and implementing ecosystem restoration the exercise often leads to projects with low probabilities of success. Restoration planners often rely on simplistic assumptions and these lead to design criteria and factors of success that are meaningless in terms of achieving desired and sustainable results and outcomes.

Complexities in predicting both "what would have been" and "what could be" and the need to demonstrate equivalency of the restored resources relative to baseline favors goals that value in-kind and proximate (local or onsite) restoration based on current and/or historic conditions over out-of-kind or remote restoration that might be more aligned with building resilience. This may result in restoration goals focused on returning resources to pre-disturbance conditions [8-10], and does not typically result in restoration that account for nonstationarity or that adds ecological resilience [11]. Establishing restoration goals accounting for non-stationarity is the critical first step in planning restoration that accounts for future uncertainty and changing conditions. This requires some important but potentially difficult policy and procedural decisions, including:

- decisions whether the analysis is focused on compensating for the affected ecosystem service or species regardless of precise location vs. focused on enhancing services provided at the specific impacted site;

- deciding how potentially conflicting goals, such as environmental justice vs. climate resilience, should be balanced;

- $\quad$ selecting the approach applied to scale restoration (e.g., HEA, or REA);

- determining how habitat connectivity and genetic diversity increase ecosystem flows;

- accommodating metrics to demonstrate equivalency since restoration may or may not be in-kind, or directly replace the same services; 
- including actions that serve long-term species resilience such as off-site or "out-of-kind" actions or restoration if future local scenarios are not projected to be conducive to restoration and species viability.

Not including the implications of non-stationarity in selecting restoration alternatives at sites where the potential level of ecosystem service of interest is declining and/or likely to change significantly biases the selection of restoration alternatives to those most closely aligned with assumptions of stationarity. Not including non-stationarity also increases the risk of restoration failure if baseline conditions are changing over time.

\subsection{Accommodate uncertainty into HEA analysis structure}

As presently used, HEA is a deterministic model with few or no stochastic elements. Temporal impacts of service loss, recovery time, and the length of time for compensatory restoration to reach full function and thereafter provide additional benefits have been assessed using a time-series of steady state events. Variability of model input parameters may be calculated using a weighted probability of an individual variable in order to report a deterministic model result. Concerns of non-stationarity in HEA analyses are often ignored because of the uncertainty with selected model inputs or the interactions between model inputs.

An alternative approach to incorporate non-stationarity is to develop a stochastic model of the expected outcomes using Bayesian networks or Monte Carlo simulation that incorporate non-stationarity drivers. The peak in the distribution of Monte Carlo model outcomes represents the best estimate of expected value. This approach more accurately summarizes the level of certainty in the analysis and helps define which model inputs and approaches have the greatest impact on the results, thus guiding future studies based on the value of information. If acceptable estimates of selected input parameters cannot be provided for a stochastic model, scenario-based approaches should be considered to address those parameters.

\subsection{Incorporate geospatial relationships to address connectivity and resilience}

Anthropogenic land use changes have a drastic impact on habitat loss and fragmentation. The heterogeneity and connectivity of habitat conditions are important measures of habitat value for both scaling of habitat losses and the selection and design for habitat restoration. HEA and most other resource and land management programs model and sum changes by categories but do not track critical relationships between habitat categories, life history requirements, and other critical ecosystem functions. Following the "what gets measured gets done" philosophy, scaling tools such as HEA need to incorporate and value applicable metrics of ecological connectivity to ensure restoration plans account for non-stationarity and adaptation goals. 
Figure 3 (modified from Hanson et al. [7]) depicts the importance of geospatial relationships in HEA. In this example significantly different post forest fire conditions with different ecosystem service and recovery values can fail to be appropriately defined using HEA. Panels $\mathrm{B}$ and $\mathrm{C}$ in fig. 3 both represent an 80 percent mortality-weighted impact following a severe forest fire compared to pre-fire conditions (Panel A). Although the impact scenarios depicted in Panels B and C could be mathematically equivalent using HEA, in reality they would have significantly different ecological value and recovery curves. Similar types of challenges can arise when using HEA to scale habitat restoration in long stretches of aquatic systems such as rivers.

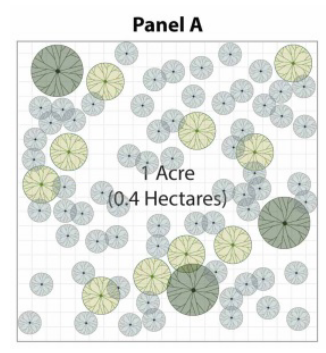

Figure 3: Example geospatial relationships in HEA.

When restoration is required to compensate for ecological resource losses or damages, it is a simple economic reality that liable parties will seek the most cost effective-restoration projects that will satisfy restoration goals or mitigation requirements. Table 1 addresses metrics and incentives for desired outcomes and objectives because what gets measured, gets done. The table provides a few examples on how connected habitats and ecosystem services might be valued. If analysts using HEA are unable to identify service loss indicators which incorporate an empirical measure of connectivity in the indicators for habitat value, an alternative approach is to create multiple subcategories within each habitat type with similar subjective ratings of connectivity. As a last resort for complex settings, regulators might consider incorporating policy decisions in the restoration scaling that subjectively acknowledge difficult to quantify, but undeniably different, expected values.

\subsection{Align discounting with building resilience}

With HEA, restoration scaling incorporates a discount rate to bring past ecosystem losses and future gains to a net present value in order to scale restoration based on the assumed preference towards having a restoration project restore function in the present rather than sometime in the future. Dunford et al. [12] and the US Environmental Protection Agency [13] provide an analysis of the theory behind the selection of an appropriate discount rate for evaluating restoration requirements of environmental damages. US trustee agencies have 
established policies requiring a uniform discount rate in determining compensatory restoration at NRDA sites. Restoration at sites which have long, multigenerational recovery periods and/or where baseline conditions are subject to significant levels of non-stationarity may result in: (1) benefits accruing to one generation while costs accrue to another, and/or (2) potential benefits from building resilience being excessively discounted. Such scenarios would be inconsistent with the assumptions used to establish policies regarding discount rates at NRDA sites and warrant a more thorough analysis.

Table 1: $\quad$ Metrics and incentives.

\begin{tabular}{|l|l|l|}
\hline Habitat type & Non-stationarity value & Possible metric or credit \\
\hline Seagrass & $\begin{array}{l}\text { Provides wave attenuation, } \\
\text { reduces coastal erosion and } \\
\text { storm surge }\end{array}$ & $\begin{array}{l}\text { Restored area plus portion of } \\
\text { habitat values for area protected } \\
\text { by seagrass }\end{array}$ \\
\hline Riverine & $\begin{array}{l}\text { Off-channel resting in high } \\
\text { velocity urban rivers with } \\
\text { limited habitat }\end{array}$ & $\begin{array}{l}\text { Incentivized restoration in higher } \\
\text { developed areas based on } \\
\text { ecological value }\end{array}$ \\
\hline Upland & Habitat connectivity corridor & $\begin{array}{l}\text { Restored area plus a multiplier for } \\
\text { newly connected areas }\end{array}$ \\
\hline Riparian & $\begin{array}{l}\text { Widen, add gradual grade to } \\
\text { allow future water levels and } \\
\text { flood storage }\end{array}$ & $\begin{array}{l}\text { Compound interest to value the } \\
\text { spatial flexibility; multiplier to } \\
\text { value flood storage }\end{array}$ \\
\hline
\end{tabular}

\subsection{Climate proof restoration plans and embrace restoration portfolios}

A key approach for managing trajectories of change is to build ecological resilience. Climate-proofing describes measures take to increase the resilience of restoration projects in the face of climate change. Chapin et al. [14] define resilience as the capacity of a system "to absorb a spectrum of shocks or perturbations and to sustain and develop its fundamental function, structure, identity and feedbacks as a result of recovery or reorganization in a new context." Although environmental liabilities are defined by the need to restore conditions to the pre-perturbation baseline, there is an implicit assumption that the pre-disturbance baseline was stationary and sustainable. Chapin et al. identify three broadly overlapping sustainability approaches that are illustrated in fig. 4:

- $\quad$ Reducing vulnerability to expected changes.

- Fostering resilience to sustain desirable conditions in the face of perturbations and uncertainty.

- $\quad$ Transforming from undesirable trajectories when opportunities emerge.

Climate-proofing and adaptation can contribute to all three approaches. However, transforming from an undesirable trajectory and including sustainability in the restoration goals may run counter to existing regulations, policies, and guidance. Policy studies identifying barriers and opportunities to integrate restoration planning with building resilience are recommended. 


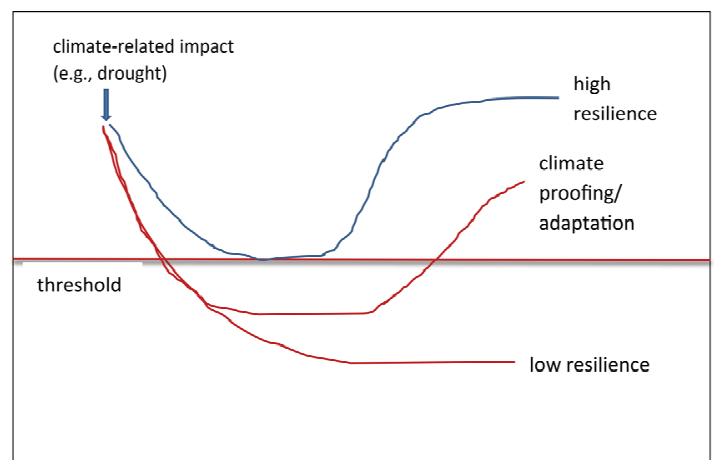

Figure 4: Climate proofing to achieve sustainable restoration design despite climate related impacts.

\section{Conclusion}

Given the increasing evidence of non-stationarity, it is essential that restoration planning develop approaches to address the changing variance in ecosystem conditions. In NRDA's case, this creates significant need and opportunity for fundamental changes in existing or new methods accommodating changing baselines, ecosystem trajectories, and uncertainties as restoration progresses. The first challenge is defining how non-stationary baselines may fluctuate and then scaling restoration to potential future scenarios under changing and uncertain conditions. Another challenge is to consider the legal and policy issues around designing restoration based on potential future, rather than current actual, conditions as well as climate adaptation and resilience goals. Rather than recovery to a "prior condition" baseline, the goal may become building resilience and the capacity of the ecosystem to sustain fundamental structures, processes, and functions in the face of increasing variability. In this context, monitoring and adaptive management of restoration will take on increasing importance, and a key challenge will be how to adjust regulatory decision-making to account for non-stationarity.

This paper's recommendations are meant as a starting point for adapting and developing new methods and guidance to align HEA with adaptation strategies for non-stationarity. Even if these recommendations are fully developed, nonstationarity by definition represents significant risks to restoration plans and it may not be possible to fully address non-stationarity on a site-by-site basis. As a result, creating a portfolio of restoration efforts that satisfy short-term primary and near-term compensatory restoration needs while also including restoration projects that incorporate long-term trajectories may prove a robust strategy to further hedge against risks and develop sustainable restoration. 


\section{References}

[1] Milly, P.C.D. et al. Stationarity is dead. Whither water management? Science 319(5863): 573-574, 2008.

[2] National Fish, Wildlife and Plants Climate Adaptation Partnership. National Fish, Wildlife and Plants Adaptation Strategy. Association of Fish and Wildlife Agencies, Great Lakes Indian Fish and Wildlife Commission, NOAA, USFWS, 2012.

[3] Vermeulen, S.J. et al. Addressing uncertainty in adaptation planning for agriculture. Proceedings of the National Academy of Sciences 110(21): 8357-8362, 2013.

[4] Kimball, S.S. Forest Fire Damages in Transition. The Federal Lawyer, 3846, 2009.

[5] SWCA. Habitat Equivalency Analysis for mitigation of the Gateway West Transmission Line. Prepared for the Bureau of Land Management, US Fish and Wildlife Service, Wyoming Game and Fish Department, Idaho Fish and Game Department, Rocky Mountain Power, and Idaho Power Company, 2012.

[6] Rohr, J.R. et al. Implications of global climate change for natural resource damage assessment, restoration, and rehabilitation. Environmental Toxicology and Chemistry 32(1): 93-101, 2013.

[7] Hanson, D.A., E.M. Britney, C.J. Earle and T.G. Stewart. Adapting habitat equivalency analysis (HEA) to assess environmental loss and compensatory restoration following severe forest fires. Exploring the Mega-Fire Reality - Forest Ecology and Management Special Issue: The Mega-Fire Reality, 294: 166-177, 2013.

[8] White, P.S., and J.L. Walker. Approximating nature's variation: Selecting and using reference information in restoration ecology. Restoration Ecology 5: 338-349, 1997.

[9] Swetnam, T.W., C.D. Allen, and J.L. Betancourt. Applied historical ecology: Using the past to manage for the future. Ecological Applications 9: 1189-1206. 1999.

[10] Egan, D., and E.A. Howell, editors. The Historical ecology handbook: A restorationist's guide to reference ecosystems. Island Press, Washington, D.C. 2001.

[11] Harris, James A., Hobbs, Richard J., Higgs, Eric, and Aronson, James. Ecological restoration and global climate change. Restoration Ecology Vol. 14, No. 2, pp. 170-176 June, 2006.

[12] Dunford, R.W., T.C. Ginn, and W.H. Desvousges. The use of habitat equivalency analysis in natural resource damage assessments. Ecological Economics 48, 49-70, 2004.

[13] US Environmental Protection Agency. Guidelines for preparing economic analysis. EPA-240-R-10-001, 2010.

[14] Chapin, F.S. III et al. Ecosystem stewardship: sustainability strategies for a rapidly changing world. Trends in Ecology and Evolution, 25: 241-249, 2009. 